\title{
APPLICATIONS AND EXTENSIONS OF GOURSAT NORMAL FORM TO CONTROL OF NONLINEAR SYSTEMS
}

\author{
RICHARD M. MURRAY
}

Division of Engineering and Applied Science

California Institute of Technology

Pasadena, California 91125

murray@design.caltech.edu

\section{Abstract}

The Goursat normal form theorem gives conditions under which an Pfaffian exterior differential system is equivalent to a certain normal form. This paper details how the Goursat normal form and its extensions provide a unified framework for understanding feedback linearization, chained form, and differential flatness.

\section{INTRODUCTION}

Traditionally, geometric nonlinear control theory has relied on the vector field interpretation of affine nonlinear control systems to provide a set of tools for studying the structural properties of nonlinear systems. In particular, properties such as accessibility, observability, and feedback linearizability can be formulated in terms of involutivity or rank conditions on certain distributions which are constructed from the description of the system. An overview of these tools can be found in Isidori [9] and other texts.

Recently, there has been an increased interest in alternative formulations of the control problem, where control systems are modelled using one-forms instead of vector fields. Systems which are described using one-forms are a subset of a class of systems known as exterior differential systems. Although one-forms and vector fields are dual to one another, there are tools available in theory of exterior differential systems which have no simple counterpart in the traditional geometric framework.

One of the main theorems which links many of the results available in the study of control systems via exterior differential forms is the Goursat Normal Form theorem. It gives a set of explicit conditions under which a codistribution on a manifold admits a local basis in a certain normal form. As we shall show in the sequel, this theorem can be applied to wide variety of problems in nonlinear control theory, including problems in determining a nilpotent basis for a given distribution, converting nonholonomic control systems into chained form, and feedback linearization using static state feedback. In this paper we present a survey of these various results and establish some connections between work in control of nonholonomic systems, feedback linearizability, and differential flatness.

\section{Mathematical PRELIMINARIES}

2.1. Pfaffian Systems. The results in this paper make extensive use of tools from the theory of exterior differential systems. We present a brief overview of the relevant concepts here. A detailed description can be found in the monograph by Bryant et al. [2] or the dissertation by Sluis [15]

Let $M$ be a manifold of dimension $n$ with cotangent bundle $T^{*} M$; let $\Omega^{p}(M)$ denote the set of smooth exterior $p$-forms on $M$. Define $\Omega^{*}(M)$ as the set of smooth exterior differential forms of all orders on a manifold $M$, $\Omega^{*}(M)=\bigoplus \Omega^{p}(M)$. An exterior differential system is given by an ideal $\mathcal{I} \subset \Omega^{*}(M)$ that is closed under exterior differentiation.

A Pfaffian systern is an exterior differential system which is generated by a set of linearly independent oneforms

$$
I=\left\{\alpha^{1}, \ldots, \alpha^{8}\right\} .
$$

The associated ideal has the form

$$
\mathcal{I}=\left\{\omega \wedge \theta: \omega \in I, \theta \in \Omega^{*}(M)\right\} .
$$

We call $s$ the dimension of the system (assumed constant)

The exterior derivative induces a mapping $\delta: I \rightarrow$ $\Omega^{2}(M) / I$ :

$$
\delta: \lambda \mapsto d \lambda \bmod I \in \Omega^{2}(M) .
$$

The mapping $\delta$ is a linear mapping over the ring of smooth functions on $M, C^{\infty}(M)$ :

$$
\begin{aligned}
\delta(f \alpha+g \beta) & =d f \wedge \alpha+f d \alpha+d g \wedge \beta+f d \beta \bmod I \\
& =f d \alpha+g d \beta \bmod I \\
& =f \delta(\alpha)+g \delta(\beta)
\end{aligned}
$$

It follows that the kernel of $\delta$ is a subbundle of $\Omega(M)$ (i.e., at each point $p \in M$, the kernel of $\delta$ is a linear subspace of $\left.T_{p}^{*} M\right)$. We call this subbundle $I^{(1)}$, the first derived system of $I$ :

$$
I^{(1)}=\operatorname{ker} \delta=\{\lambda \in I: d \lambda \bmod I \equiv 0\} .
$$

We represent $I^{(1)}$ using a basis of one-forms and hence $I^{(1)}$ also generates a Pfaffian system.

Since $I^{(1)}$ is itself a codistribution on $M$, we can continue this construction and generate a filtration

$$
I=I^{(0)} \supset I^{(1)} \supset \cdots \supset I^{(N)} .
$$

If the dimension of each $I^{(i)}$ is constant, then this construction terminates for some finite integer $N$. In this case, we call equation (1) the derived flag of $I$ and $N$ the derived length.

The derived flag describes the integrability properties of the ideal generated by $I$. If $I$ is completely integrable, 
then by Frobenius's theorem we have $I^{(1)}=I^{(0)}$, i.e. the derived length of the flag is zero. In fact, $I^{(N)}$ is always integrable since by definition $d I^{(N)} \bmod I^{(N)} \equiv 0 . I^{(N)}$ is the largest integrable subsystem contained in $I$. Thus if $I^{(N)}$ is not empty, then there exist functions $h_{1}, \ldots, h_{r}$ such that $\left\{d h_{i}\right\} \subset\{I\}$. In the context of control theory, this means that the system is not controllable since there exist algebraic functions which provide a foliation of the state space and it is impossible to move from one leaf of the foliation to another. The converse of this controllability result is provided by the following version of Chow's Theorem:

Theorem 1 (Chow). Let $I=\left\{\alpha^{1}, \ldots, \alpha^{*}\right\}$ represent a set of constraints and assume that the derived flag of the system exists. Then, there exists a path $x(t)$ between any two points satisfying $\alpha^{i}(x) \dot{x}=0$ for all $i$ if and only if there exists an $N$ such that $I^{(N)}=0$.

When doing computations with exterior differential systems, it is convenient to choose a basis of one-forms whose structure matches that of the derived flag. We say that a basis $\left\{\alpha^{i}\right\}$ is adapted to the derived flag if

$$
I^{(i)}=\left\{\alpha^{2}, \ldots, \alpha^{s_{i}}\right\},
$$

where $s_{i}$ is a strictly decreasing sequence of integers. In other words, an adapted basis is one in which the derived systems are calculated by dropping elements from the end of the basis. An adapted basis can be calculated by computing the derived flag and then choosing the basis elements starting with a basis for $I^{(N-1)}$ and proceeding backwards.

2.2. Goursat normal form. The main results of this paper are a direct consequence of the following theorem, whose proof can be found in Bryant et al. [2, pp. 54-57].

Theorem 2. Goursat normal form

Let $U$ be an open subset of $\mathbb{R}^{n}$ and $I=\left\{\alpha_{1}, \ldots, \alpha_{0}\right\}$ be a collection of smooth, linearly independent one-forms defined on $U$. If there exists a one-form $\pi \neq 0 \bmod I$ such that

$$
\begin{aligned}
d \alpha_{i} & \equiv-\alpha_{i+1} \wedge \pi \bmod \alpha_{1}, \ldots, \alpha_{i} \quad i=1, \ldots, s-1 \\
d \alpha_{s} & \neq 0 \bmod I
\end{aligned}
$$

then there exists a set of coordinates $x$ such that

$$
I=\left\{d x_{n}-x_{n-1} d x_{1}, \ldots, d x_{3}-x_{2} d x_{1}\right\} .
$$

A few comments on the statement of this theorem are in order. The conditions of the theorem require the existence of a special basis $\left\{\alpha^{i}\right\}$ and a special one-form $\pi$. A quick calculation shows that the basis $\left\{\alpha^{i}\right\}$ is adapted to the derived flag of the system and hence if we start with an adapted basis, the real requirement is the existence of a one-form $\pi$ which satisfies the congruences. Determining $\pi$ can involve a further scaling of the adapted basis which preserves the adapted structure. For most examples, $\pi$ can be determined by a combination of physical insight and repeated guessing.
The Goursat congruences are somewhat unsatisfying since they require the existence of a one-form $\pi$. Necessary and sufficient conditions for the existence of such $a \pi$, and hence for converting a set of constraints into Goursat normal form, were presented in [11]. We summarize the main result here.

Let $I=\operatorname{span}\left\{\omega^{1}, \ldots, \omega^{2}\right\}$ be a codistribution on $\mathbb{R}^{n}$ and write $\Delta=I^{\perp}$ for the distribution which spans the null space of the codistribution. We define two nested sets of distributions:

$$
\begin{aligned}
E_{0} & =\Delta & F_{0} & =\Delta \\
E_{1} & =E_{0}+\left[E_{0}, E_{0}\right] & F_{1} & =F_{0}+\left[F_{0}, F_{0}\right] \\
E_{2} & =E_{1}+\left[E_{1}, E_{1}\right] & F_{2} & =F_{1}+\left[F_{1}, F_{0}\right] \\
& \vdots & & \vdots \\
E_{i+1} & =E_{i}+\left[E_{i}, E_{i}\right] & F_{i+1} & =F_{i}+\left[F_{i}, F_{0}\right] .
\end{aligned}
$$

Under the assumption that each distribution is constant rank, the two sequences have finite length (possibly different).

The filtration $\left\{F_{i}\right\}$ is the the one which usually appears in the context of nonlinear controllability and feedback linearization. In particular, $F_{i}$ consists of all brackets up to order $i$. The distribution $E_{i}$ also contains all brackets of order $i$, but may contain additional Lie products of higher order. This is due to the recursive construction of $E_{i}$, as opposed to the iterative construction of $F_{i}$. The filtration $E_{i}$ is precisely the sequence of distributions which is perpendicular to the derived flag of $I=\Delta^{\perp}$.

Theorem 3 ([11]). Given a 2-dimensional distribution $\Delta=I^{\perp}$ such that

$$
\operatorname{dim} E_{i}=\operatorname{dim} F_{i}=i+2 \quad i=0, \ldots, n-2,
$$

in a neighborhood of the origin, there exists a one-form $\pi$ and $a$ basis $\left\{\alpha^{1}, \ldots, \alpha^{s}\right\}$ for $I$ which satisfy the Goursat congruences in that same neighborhood.

This theorem allows us to completely characterize all exterior differential systems of codimension 2 are locally equivalent to a system in Goursat form.

The fact that the dimension of the derived flag (or equivalently the filtration $\left\{E_{i}\right\}$ ) is not sufficient to guarantee equivalence to the Goursat normal form has been pointed out by Giaro, Kumpera, and Ruiz [7], who constructed a counterexample in 5 dimensions. They also note that if $\operatorname{dim} E_{i}=i+2$ then the Goursat conditions hold on an open dense set. This result has also been pointed out by Martin and Rouchon [10] in the context of linearization of driftless systems. For the applications considered here, we desire a transformations which hold on a neighborhood and hence the stronger result of Theorem 3 is needed.

\section{Applications of the Goursat normal form THEOREM}

3.1. Nonholonomic control problems. Consider the problem of controlling a mechanical system with $n$ dimensional configuration space $Q$ subject to a set of independent kinematic constraints having the form:

$$
\left\langle\omega_{i}(q), \dot{q}>=0 \quad i=1, \ldots, k .\right.
$$


The $\omega_{i}$ 's are a basis for a codistribution on $Q$ which restricts the velocity of the system to be zero in certain directions. This class of constraints often arises in mechanical systems in which rolling is present. Examples include mobile robots, whose wheels are allowed to roll and spin but not slide sideways, and multi-fingered robot hands, where two surfaces (the fingertip and the object) roll against each other without slipping. The basic control problem associated with this class of systems is to find a path connecting two given points such that the constraints (4) are satisfied at all times. We refer to this as the trajectory generation problem.

The set of kinematic constraints (4) is holonomic if the constraints restrict the motion of the system to a manifold of dimension $n-k$. In this case, the constraints on the system can be rewritten as a set of algebraic constraints on the configuration variables $q$. The constraints are nonholonomic if they do not constrain the system to lie on a submanifold of the dimension $n-k$. In particular, we are most interested in constraints which are completely nonholonomic: any point in the configuration space can be reached via an admissible trajectory. If the constraints are not completely nonholonomic, the system can still be analyzed by restricting the initial and final configurations to lie on the same integrable submanifold.

The use of exterior differential systems, and in particular Pfaffian systems, to study nonholonomic problems is quite natural. By Chow's theorem, a set of nonholonomic constraints is completely nonholonomic if and only if the bottom derived system satisfies $I^{(N)}=\{0\}$. Although this answers the problem of whether a path between any two points exist, it does not help us find that that path.

In terms of vector fields, one can formulate the trajectory generation problem using a set of vector fields $\left\{g_{1}, \ldots, g_{m}\right\}, m=n-k$, which form a basis for the distribution annihilated by the constraints. The construction of a path satisfying the constraints is then equivalent to finding an input $u(t) \in \mathbb{R}^{m}$ such that the system

$$
\dot{q}=g_{1}(q) u_{i}+\ldots g_{m}(q) u_{m}
$$

is steered from $q(0)=q_{0}$ to $q(T)=q_{1}$ for some $T>0$.

One tool which has proved useful in the study of nonholonomic control problems is a certain normal form called "chained form" [13]. The simplest case of system in chained form is given by

$$
\begin{aligned}
\dot{x}_{1} & =u_{1} \\
\dot{x}_{2} & =u_{2} \\
\dot{x}_{3} & =x_{2} u_{1} \\
\dot{x}_{4} & =x_{3} u_{1} \\
\vdots & \\
\dot{x}_{n} & =x_{n-1} u_{1}
\end{aligned}
$$

A simple calculation shows that this set of vector fields is perpendicular to the one-forms of the Goursat normal form, and hence the Goursat normal form can be used to give conditions under which a system is feedback transformable to a system in chained form. The following proposition follows immediately by application of Theorems 2 and 3.
Proposition 4. There exists a feedback transformation which puts a two-input nonholonomic system into chained form (5) if and only if

$$
\operatorname{dim} E_{i}=\operatorname{dim} F_{i}=i+2 \quad i=0, \ldots, n-2 .
$$

Thus, the class of two-input systems which can be converted into chained form (with a single chain) is completely characterized by an algebraic test. It is interesting to note that although chained systems are not generic, they occur frequently in applications. For example, all controllable, two-input, regular nonholonomic systems in 3 and 4 dimensions are locally feedback equivalent to a system in chained form. This follows from Darboux's theorem and Engel's theorem, respectively, and is a special case of the results presented above (see [2, pp. 50-51] for a proof of Engel's theorem). These special cases have also pointed out by Hermes [8]. Other systems, such as a car with 1 or 2 trailers also satisfy the conditions necessary to put them into chained form. In fact, it has recently been show that a car with $N$ trailers can be converted into chained form $[16,14,17]$.

3.2. Feedback linearization. Systems in chained form are closely related to systems in Brunovsky canonical form. If we ignore the first line of equation (5) and set $u_{1}=1$, we get a chain of integrators driven by $u_{2}$. Based on this, one might expect that the conditions for converting a system into chained form could be rewritten in terms of involutivity of certain distributions, corresponding to the feedback linearizability conditions. Sufficient conditions for converting a system into chained form using precisely this idea were presented in [13] and [4].

A natural question is whether the Goursat conditions can be used to characterize systems which are feedback linearizable via static state feedback. As a first attempt, one might do the following: Let $f$ and $g$ be vector ficlds on a manifold $M$ which describe an affine nonlinear system. Construct an associated system on $\mathbb{R} \times M$ with the vector fields

$$
g_{1}=\left[\begin{array}{l}
1 \\
f
\end{array}\right] \quad g_{2}=\left[\begin{array}{l}
0 \\
g
\end{array}\right] .
$$

If the original system is feedback linearizable then $g_{1}$ and $g_{2}$ can be converted into chained form. However, the converse is not true, as the following example shows.

Example 1. (contributed by Michiel van Nieuwstadt) Consider the affine control system

$$
\begin{aligned}
& \dot{x}_{1}=-x_{1}+u \\
& \dot{x}_{2}=x_{2} u \\
& \dot{x}_{3}=x_{3} u
\end{aligned} \quad \Longrightarrow \quad f=\left[\begin{array}{c}
-x_{1} \\
0 \\
0
\end{array}\right], \quad g=\left[\begin{array}{c}
1 \\
1+x_{2} \\
1+x_{3}
\end{array}\right]
$$

It is easy to verify that this system is accessible but not not feedback linearizable (its linearization is not controllable). It can also be verified that the augmented system

$$
g_{1}=\left[\begin{array}{c}
1 \\
-x_{1} \\
0 \\
0
\end{array}\right] \quad g_{2}=\left[\begin{array}{c}
0 \\
1 \\
1+x_{2} \\
1+x_{3}
\end{array}\right]
$$

satisfies the rank conditions of Theorem 3 and hence can be converted into chained form. This involves switching the roles of $g_{1}$ and $g_{2}$. 
The proper application of the Goursat theorem to study feedback linearization was developed by Gardner and Shadwick for the case of MIMO feedback linearization via static state feedback [6]. For the single input, single output case, there criterion can be stated as follows. Let $I$ be a codistribution on $\mathbb{R}^{n} \times \mathbb{R} \times \mathbb{R}^{+}$given by

$$
\alpha_{i}=d x_{i}-f_{i}(x) d t-g_{i}(x) u d t \quad i=1, \ldots, n .
$$

This defines a Pfaffian system with codimension 2.

Theorem 5. An affine nonlinear control system is feedback linearizable via static state feedback if and only if there exists a basis for the associated Pfaffian system (7) which satisfies the Goursat congruences with $\pi=d t$.

The difference between the necessary and sufficient conditions for converting a nonholonomic system to chained form and those for feedback linearization are in the extra freedom of choosing $\pi$ in the nonholonomic case. A further complication which arises in the proof of Theorem 5 is the possibility that the feedbeck transformation which converts the system into Goursat form might depend on time or depend nonlinearly on the inputs. However, the fact that the original system is autonomous and affine allows one to show that this does not occur (see [6] for details).

The lack of freedom in choosing $\pi$ implies that, although they are very similar, chained (or Goursat) form and Brunovsky form are not completely equivalent. Thus, the lack of necessary and sufficient conditions for converting a system into chained form using involutivity of certain distributions is perhaps not surprising. However, the search for such conditions remains an interesting problem.

\section{EXTENDED GOURSAT NORMAL FORM}

It is possible to extend the Goursat normal form theorem to handle cases other than codimension 2. This was done by Gardner and Shadwick in the context of feedback linearization [6]. We present here a modified version of their results and discuss its applications.

Let $U$ be an open subset of $\mathbb{R}^{n}$ and $I=\left\{\alpha_{1}^{j}, \ldots, \alpha_{s_{j}}^{j}\right.$ : $j=1, \ldots, m-1\}$ be a collection of smooth, linearly independent one-forms defined on $U$. Let $I^{(i)}$ denote the $i^{\text {th }}$ derived system of $I$ which we take as

$$
I^{(i)}=\left\{\alpha_{k}^{j}: j=1, \ldots, m-1, k=1, \ldots, s_{j}-i\right\},
$$

where $s_{1} \geq s_{2} \geq \cdots \geq s_{m-1}>0$.

We make use of the following version of Frobenius's theorem [6]:

Theorem 6. Let $\omega_{1}, \ldots, \omega_{p}$ be a set of linearly independent 1 -forms and let $f_{1}, \ldots, f_{m}$ be functions whose diffenentials are linearly independent of each other and the 1 -forms $\left\{\omega_{i}\right\}$. If $\omega$ and $f$ satisfy the relative Frobenius condition

$d \omega_{i} \wedge \omega_{1} \wedge \cdots \wedge \omega_{p} \wedge d f_{1} \wedge \cdots \wedge d f_{m}=0 \quad i=1, \ldots, p$

then there exist coordinate functions $z_{1}, \ldots, z_{p}$ and coefficient functions $a_{i j}$ and $b_{i j}$ such that

$$
\omega_{i}=a_{i j} d z_{j}+b_{i j} d f_{j} .
$$

Proof. Apply the standard Frobenius theorem (forms version) to the codistribution $\left\{\omega_{1}, \ldots, \omega_{p}, d f_{1}, \ldots, d f_{m}\right\}$.

Theorem 7. Extended Goursat normal form

If there exists a single one-form $\pi \neq 0$ such that for all $j$

$$
\begin{aligned}
& d \alpha_{i}^{j} \equiv-\alpha_{i+1}^{j} \wedge \pi \bmod \Gamma^{\left(\iota_{j}-i\right)} \quad i=1, \ldots, s_{j}-1 \\
& d \alpha_{s_{j}}^{j} \neq 0 \quad \bmod I
\end{aligned}
$$

then there exists a set of coordinates $x \in \mathbb{R}^{s_{1}+\cdots+s_{m-1}+m}$ such that

$I=\left\{d x_{1}^{j}-x_{2}^{j} d x_{0}, \ldots, d x_{s_{j}}^{j}-x_{s_{j+1}}^{j} d x_{0}: j=1, \ldots, m-1\right\}$.

Sketch of proof. To simplify the exposition, it will be convenient to use slightly different notation and consider only the case where $m=3$. We assume that the exterior system consists of the forms

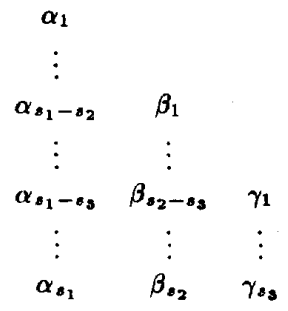

Without loss of generality we take this basis to be adapted to the derived flag and hence $I^{(1)}$ consists of all forms except those on the bottom row, $I^{(2)}$ consists of all forms except those on the bottom two rows, etc. We wish to show that there exists a choice of coordinates $q=(x, y, z, t)$ such that

$$
\begin{array}{rl}
\alpha_{i}=d x_{i}-x_{i+1} d t & i=1, \ldots, s_{1} \\
\beta_{i}=d y_{i}-y_{i+1} d t & i=1, \ldots, s_{2} \\
\gamma_{i}=d z_{i}-z_{i+1} d t & i=1, \ldots, s_{3}
\end{array}
$$

Here $t$ is not necessarily the variable associated with time, although in certain cases (e.g. feedback linearization) it does play this role.

We consider first the case where $s_{1}>s_{2}>s_{3}$ and hence the length of each chain is distinct. Define $s_{12}=$ $s_{1}-s_{2}$. The coordinates for $t$ and $x_{1}$ through $x_{s_{12}+1}$ are guaranteed exactly as in the proof of the standard Goursat form. It also follows from this construction that $\pi$ can be taken to be $d t$. The next level of congruences gives

$$
\left.\begin{array}{rl}
d \alpha_{s_{12}} & \equiv \alpha_{s_{12}+1} \wedge d t \\
d \beta_{1} & \equiv \beta_{2} \wedge d t
\end{array}\right\} \quad \bmod \alpha_{1}, \ldots, \alpha_{s_{12}}, \beta_{1} .
$$

Since $\alpha_{s_{12}}=d x_{s_{12}}-x_{s_{12}+1} d t$ we have that

$$
\alpha_{s_{12+1}} \wedge d t \wedge d x_{s_{12+1}} \equiv 0 \bmod \alpha_{1}, \ldots, \alpha_{s_{12}}, \beta_{1}
$$

and, after redefining $\alpha_{\boldsymbol{\theta}_{12}+1}$ to convert the congruence to an equality, it follows that

$$
\alpha_{s_{12}+1}=a d x_{s_{12}+1}+b d t .
$$

The coefficient $a$ cannot be equal to zero and hence we can scale $\alpha_{s_{12}+1}$ and define $x_{a_{12}+2}=-b / a$. 
In the other tower, we have that

$$
d \beta_{1} \wedge \beta_{1} \wedge d t=0
$$

and it follows from the relative Frobenius theorem that there exist coordinates such that

$$
\beta_{1}=a d y_{1}+b d t \text {. }
$$

Setting $y_{2}=-b / a$ and scaling $\beta_{1}$ gives the desired form. The rest of the $y$ coordinates follow exactly as in the Goursat case. The $z$ coordinates are computed in exactly the same way using $\gamma_{i}$.

In the case that $s_{2}=s_{3}$ we have two towers entering the calculation at the same time. However, it is still true that

$$
\begin{aligned}
& d \beta_{1} \wedge \beta_{1} \wedge \gamma_{1} \wedge d t=0 \\
& d \gamma_{1} \wedge \beta_{1} \wedge \gamma_{1} \wedge d t=0
\end{aligned}
$$

and so by Frobenius's theorem we can find $y_{1}$ and $z_{1}$ such that

$$
\left[\begin{array}{l}
\beta_{1} \\
\gamma_{1}
\end{array}\right]=A\left[\begin{array}{l}
d y_{1} \\
d z_{1}
\end{array}\right]+B d t
$$

where $A(q) \in \mathbb{R}^{2 \times 2}$ and $B(q) \in \mathbb{R}^{2}$. By taking linear combinations of the constraints we can eliminate the factor of $A$ to obtain

$$
\left[\begin{array}{l}
\beta_{1} \\
\gamma_{1}
\end{array}\right]=\left[\begin{array}{l}
d y_{1} \\
d z_{1}
\end{array}\right]+A^{-1} B d t .
$$

The remainder of the coordinates follow algebraically.

This version of the theorem has direct relevance to converting nonholonomic systems with more than two inputs into chained form. For example, it shows that a mobile robot consisting of a steerable car and a steerable trailer can be converted into a 3-input version of chained form. An algorithmic description of how this theorem can used for such problems is given in [3].

For feedback linearization problems, one must again set $\pi=d t$ and verify that although $u \in \mathbb{R}^{m}$ and $t \in \mathbb{R}$ are allowed in the diffeomorphisms to convert to Goursat form, the final transformation is a static state feedback transformation depending affinely on $u$. These details are verified in [6].

\section{Discussion}

5.1. Single generator systems. One of the main limitations of the use of Goursat normal form, and its extensions, is the reliance on a single one-form $\pi$ in the Goursat congruences. This limitation implies that the only control systems which can be studied using the Goursat normal form are those in which controllability is generated by a single vector field. In other words, the control Lie algebra for Goursat systems is spanned by the input vector fields along with vector fields of the form $\operatorname{ad}_{f}^{k} g_{i}$, or $\operatorname{ad}_{g_{1}}^{k} g_{i}$ in the case of nonholonomic systems. We refer to systems in which controllability can be generated by a single vector field as single generator systems. In the case of affine nonlinear systems, $f$ is predetermined, as is $\pi$. For nonholonomic systems, the Goursat theorem helps determine the $\pi$ and through it the generator $g_{1}$, which may be a pointwise linear combination of the original vector fields.
While this class of systems plays a important role in such areas as feedback linearization and mobile robotics (where the forward motion of the vehicle corresponds to the generator), in many problems a single generator does not necessarily exist. One example is given by the kinematic equations of a ball rolling on a plate $[12,1]$, which can locally be modelled by the differential equation

$$
\begin{aligned}
& \dot{x}_{1}=u_{1} \\
& \dot{x}_{2}=u_{2} \\
& \dot{x}_{3}=x_{1} u_{2}-x_{2} u_{1} \\
& \dot{x}_{4}=x_{3} u_{1} \\
& \dot{x}_{5}=x_{3} u_{2} .
\end{aligned}
$$

This system is in chained form (in the general sense defined in [13]), but does not satisfy the rank conditions for converting it into chained form with a single generator. More general conditions which capture examples such as these are still needed.

5.2. Differentially flat systems. Fliess and coworkers have recently introduced the notion of a differentially flat nonlinear system $[5,14]$. Roughly speaking, a nonlinear system with $m$ inputs is said to be differentially flat if there exist $m$ "output" functions such that the state of the system and the inputs to the system depend algebraically on these output functions and a finite number of derivatives. This class of systems is very attractive because the differentially flat outputs can be used to generate feasible trajectories for the system, potentially allowing simple algorithms for nonlinear plant inversion.

Using a result of Cartan, Martin and Rouchon [10] have shown that a necessary and sufficient condition for a 2 input nonholonomic system on $\mathbb{R}^{n}$ to be differentially flat on an open and dense set is that it satisfy the rank conditions

$$
\operatorname{dim} I^{(i)}=n-2 \quad \text { or equivalently } \quad \operatorname{dim} E_{i}=i+2 .
$$

Thus a necessary and sufficient condition for a tow-input, nonholonomic system to be differentially flat is that it be convertible to chained form in an open and dense set. The extent to which stronger conditions given by Theorem 3 are necessary to extend this result to an open neighborhood are currently unknown.

More generally, one sees readily that all systems which satisfy the Goursat congruences are differentially flat by choosing as flat outputs the coordinates at the top of each of the towers, $x_{1}^{j}, j=1, \ldots, m-1$, and $x_{0}$, the function whose differential plays the role of $\pi$ in the Goursat normal form. It follows that all feedback linearizable systems are differentially flat and that all systems which can be converted into chained form with a single generator are differentially flat. Some additional special cases of this type are given in [10].

The single generator nature of the Goursat form once again limits its usefulness in characterizing differentially 
flat systems. For example, the system

$$
\begin{aligned}
& \dot{x}_{1}=u_{1} \\
& \dot{x}_{2}=x_{4} \\
& \dot{x}_{3}=x_{2} u_{1} \\
& \dot{x}_{4}=u_{2}
\end{aligned}
$$

is differentially flat (using $x_{3}$ and $x_{1}$ as outputs), but does not satisfy the Goursat congruences with a single generator $\pi$. This example is characteristic of nonholonomic systems in which torques are used as the inputs instead of velocities.

For problems with multiple generators, such as the example given in equation (9), the use of differential flatness appears to be limited. Since the system in (9) does not satisfy the rank condition in equation (10), it is not differentially flat. However, it is in chained form and can be easily be steered between any two configurations, as shown in [13].

5.3. Conclusions. The use of exterior differential systems for studying problems in control has given new insight into the use of differential geometry as a tool for understanding the structure of nonlinear systems. While many of the results available using codistributions and derived flags are already available using vector fields and distributions, there are many problems which appear to be more natural when viewed as exterior differential systems. In particular, many recent results in control of nonholonomic systems and differential flatness have relied on these tools and the renewed interest in this area will undoubtedly generate new insights and new approaches in nonlinear control theory.

Acknowledgements. I would like to thank R. Gardnex and W. Sluis of the Fields Institute for Mathematical Sciences, Ontario, Canada for many fruitful conversations on the use of exterior differential systems for studying chained systems and for pointing out the connections between Goursat normal form and chained systems. I have also benefited from numerous discussions on this material with Linda Bushnell, Shankar Sastry, and Dawn Tilbury at UC Berkeley and Michiel van Nieuwstadt and Muruhan Rathinam at Caltech.

\section{REFERENCES}

[1] R. W. Brockett and L. Dai. Non-holonomic kinematics and the role of elliptic functions in constructive controllability. In $\mathrm{Z}$. $\mathrm{Li}$ and J. F. Canny, editors, Nonholonomic Motion Planning, pages 1-22. Kluwer, 1993.

[2] R. L Bryant, S. S. Chern, R. B. Gardner, H. L. Goldschmidt, and P. A. Griffiths. Exterior Differential Systems. Springer-Verlag, 1991.

[3] L. Bushnell, D. Tilbury, and S. Sastry. Extended goursat normal forms with applications to nonholonomic motion planning. In IEEE Control and Decision Conference, 1993.

[4] L. Bushnell, D. Tilbury, and S. Sastry. Steering three-input chained form nonholonomic systems using sinusoids. In European Control Conference, pages 1432-1437, 1993.
[5] M. Fliess, J. Levine, P. Martin, and P. Rouchon. On differentially flat nonlinear systems. C. R. Acad. Sci. Paris, 315:619-624, 1992. Serie I.

[6] R. B. Gardner and W. F. Shadwick. The GS algorithm for exact linearization to Brunovsky normal form. IEEE Transactions on Automatic Control, 37(2):224-230, 1992.

[7] A. Giaro, A. Kumpera, and C. Ruiz. Sur la lecture correcte d'un résultat d'Éli Cartan. Comptes Rendus des Séances de l'Académie des Sciences, 287, Série A:241-244, 1978.

[8] H. Hermes. Distributions and the Lie algebras their bases can generate. Proceedings of the American Mathematical Society, 106(2):555-565, 1989.

[9] A. Isidori. Nonlinear Control Systems. SpringerVerlag, 2nd edition, 1989.

[10] P. Matrtin and P. Rouchon. Feedback linearization and driftless systems. CAS internal report $446, \mathrm{Cen}$ tre Automatique et Systemes, June 1993.

[11] R. M. Murray. Nilpotent bases for a class of nonintegrable distributions with applications to trajectory generation for nonholonomic systems. Technical Report CIT/CDS 92-002, California Institute of Technology, October 1992.

[12] R. M. Murray and S. S. Sastry. Grasping and manipulation using multifingered robot hands. In R. W. Brockett, editor, Robotics: Proceedings of Symposio in Applied Mathematics, Volume 41, pages 91-128. American Mathematical Society, 1990.

[13] R. M. Murray and S. S. Sastry. Nonholonomic motion planning: Steering using sinusoids. IEEE Transactions on Automatic Control, 38(5):700-716, 1993.

[14] P. Rouchon, M. Fliess, J. Levine, and P. Martin. Flatness and motion planning: the car with $n$ trailers. In European Control Conference, pages 1518$1522,1992$.

[15] W. M. Sluis. Absolute Equivalence and its Applications to Control Theory. $\mathrm{PhD}$ thesis, University of Waterloo, 1992.

[16] O. J. Sørdalen. Conversion of the kinematics of a car with $n$ trailers into a chained form. In IEEE International Conference on Robotics and Automation, pages 382-387, 1993.

[17] D. Tilbury, R. M. Murray, and S. S. Sastry. Trajectory generation for the N-trailer problem using Goursat normal form. In IEEE Control and Decision Conference, 1993. 